\title{
Hydrodynamic studies of type I X-ray bursts
}

\author{
Jordi José* \\ Dept. Física i Enginyeria Nuclear, Universitat Politècnica de Catalunya (UPC), and Institut d'Estudis \\ Espacials de Catalunya (IEEC) \\ Sor Eulàlia d'Anzizu s/n, Campus Nord UPC, Mòdul B4-B5, $2^{a}$ planta, E-08034 Barcelona, Spain \\ E-mail: jordi.jose@upc.edu
}

\section{Fermín Moreno}

Institut d'Estudis Espacials de Catalunya (IEEC)

C. Gran Capità 2-4, Ed. Nexus-201, E-08034 Barcelona, Spain

E-mail: moreno@ieec.fcr.es

$\mathrm{X}$-ray bursts are violent stellar events that take place on the $\mathrm{H} / \mathrm{He}$-rich envelopes of accreting neutron stars, and are powered by thermonuclear runaways. These cataclysmic events constitute the most frequent source of thermonuclear stellar explosions in the Galaxy (and the fourth most energetic stellar event after $\gamma$-ray bursts, supernovae, and nova outbursts). In this paper, we present new hydrodynamical models of X-ray bursts, in spherical symmetry (1-D), from the onset of accretion up to the explosion stage, with special emphasis on the main nucleosynthetic path. Current attempts to model these explosions in 2-D are also outlined.

International Symposium on Nuclear Astrophysics - Nuclei in the Cosmos - IX

CERN, Geneva, Switzerland

25-30 June, 2006

\section{${ }^{*}$ Speaker}




\section{Introduction}

Nucleosynthesis accompanying type I X-ray bursts (hereafter, XRBs) has been extensively studied by different groups. Until recently, limited nuclear reaction networks have been adopted, with endpoints around $\mathrm{Ni}$ (Taam et al. 1993, 1996; Woosley \& Weaver 1984), Se (Hanawa et al. 1983), Kr (Koike et al. 1999), or Y (Wallace \& Woosley 1981). Calculations by Wallace \& Woosley (1984) reached ${ }^{96} \mathrm{Cd}$, but through a reduced 16 nuclei network.

Detailed nucleosynthetic calculations, with extended networks, have been performed under two different approaches: one-zone models (Schatz et al. 1999, 2002 -up to Xe-, Koike et al. 2004 -up to Bi-), and 1-D hydrodynamic models (Woosley et al. 2004; Fisker et al. 2004 -up to the SnSbTe mass region-). It is worth noting that no succesful multidimensional simulation of an X-ray burst has been reported so far. Indeed, Zingale et al. (2001) studied the ignition and propagation of a thermonuclear runaway (TNR) in the accreted envelope of a neutron star in the framework of a 2-D cartesian grid, but for too extreme conditions, beyond those characteristic of an XRB.

In this work, we present preliminary nucleosynthesis results obtained through the coupling of an extended nuclear reaction network - up to Te- and a 1-D hydrodynamic code. Simulations are carried by means of a modified version of the code SHIVA, a one-dimensional, spherically symmetric, hydrodynamic code, in Lagrangian formulation, extensively used in the modeling of classical nova outbursts (José \& Hernanz 1998). For this study, we have considered a $1.4 \mathrm{M}_{\mathrm{o}}$ neutron star, with an initial luminosity $\sim 5 \mathrm{~L}_{0}$, accreting solar-like material $(\mathrm{X}=0.759, \mathrm{Y}=0.24$, $\mathrm{X}\left[{ }^{14} \mathrm{~N}\right]=10^{-3}$ ) at a constant rate of $1.7510^{-9} \mathrm{M}_{\mathrm{o}} \mathrm{yr}^{-1}$.

Energy transport is carried by radiation and convection. A time-dependent formalism for convective transport has been included whenever the characteristic convective timescale becomes larger than the integration time step. Partial mixing between adjacent convective shells is treated by means of a diffusion equation.

Reaction rates have been obtained through the Network Generator tool (NETGEN) of the Institut d'Astronomie et d'Astrophysique (ULB, Brussels). The network includes 478 nuclei linked through a net containing 2640 reactions $[(\mathrm{p}, \gamma),(\mathrm{p}, \alpha),(\alpha, \gamma), \beta$-decays and all reverse reactions, including photodisintegrations]. Details on the specific prescriptions adopted in this work are available at the NETGEN Web page, although most of the rates are based on existing compilations (NACRE, Iliadis et al., ...) In general, no neutron or electron captures have been considered in these calculations. Electron screening is taken into account.

\section{Nucleosynthesis and nuclear flows}

The early evolution of the TNR is dominated by cold CNO burning (mainly through $\left.{ }^{12} \mathrm{C}(\mathrm{p}, \gamma){ }^{13} \mathrm{~N}\left(\beta^{+}\right){ }^{13} \mathrm{C}(\mathrm{p}, \gamma){ }^{14} \mathrm{~N}\right)$. When the temperature reaches $\mathrm{T}=2 \cdot 10^{7} \mathrm{~K}$, the dominant nuclear reactions for this particular model are of the $(\mathrm{p}, \gamma)\left(49 \%\right.$ of all reactions with fluxes $>10^{-30}$ reactions $\left.\mathrm{s}^{-1} \mathrm{~cm}^{-3}\right),(\mathrm{p}, \alpha)(10 \%)$, and $\beta^{+}(25 \%)$. At this stage, the main nuclear flow (see snapshots in Fig. 1) proceeds close to the valley of stability. 

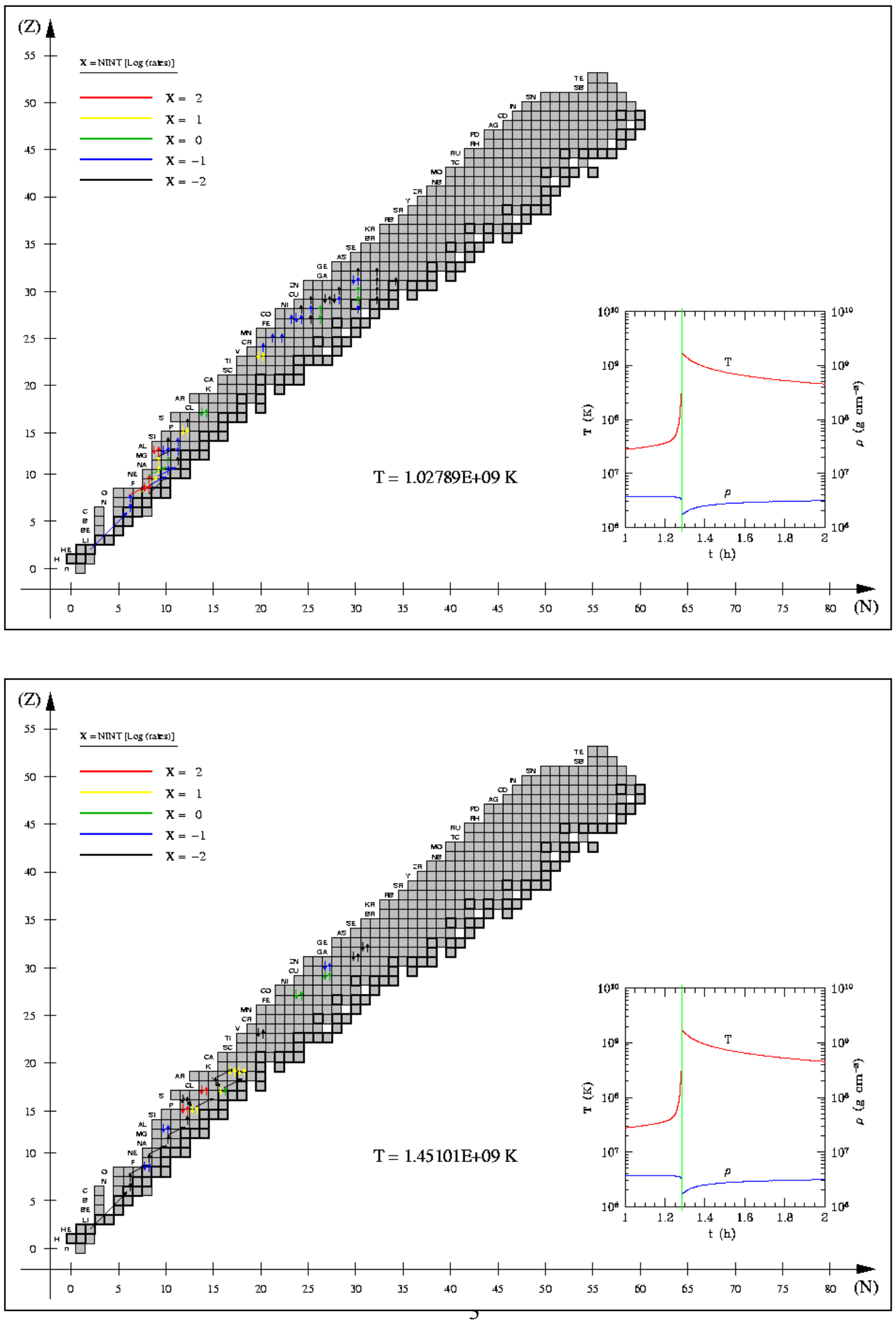


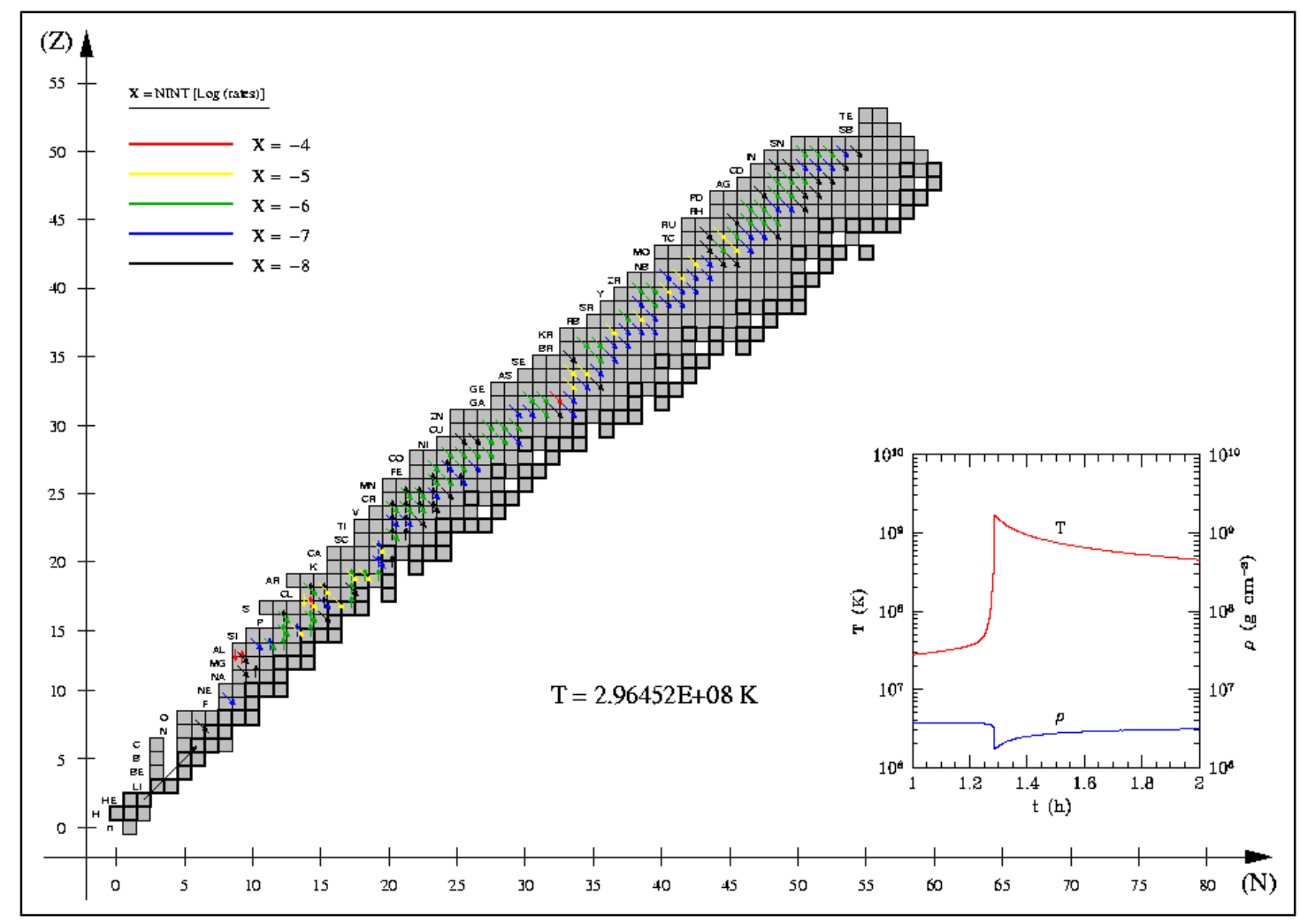

Fig. 1: Main nuclear reaction fluxes (number of reactions per unit volume and time) at different stages of the TNR during a typical X-ray burst.

When $\mathrm{T}=2 \cdot 10^{8} \mathrm{~K}$ : the nuclear activity already reaches ${ }^{40} \mathrm{Ca}$. The most abundant species (Fig. 2), with the exception of $\mathrm{H}$ and ${ }^{4} \mathrm{He}$, are now ${ }^{14,15} \mathrm{O}$. The dominant reactions are still (p, $\gamma$ ) $(40 \%)$ and $\beta^{+}$decays $(29 \%)$, but $17 \%$ are already $\alpha$-capture reactions. Shortly after, when $\mathrm{T}=2.7 \cdot 10^{8} \mathrm{~K}$, the $3 \alpha$ reaction dominates the nuclear flow, together with a suite of $(\mathrm{p}, \gamma)$ and $(\mathrm{p}, \alpha)$ reactions, some $\beta^{+}$decays (mainly ${ }^{32,33} \mathrm{Cl}$ ) and the strong photodisintegration reaction ${ }^{22} \mathrm{Al}(\gamma, \mathrm{p})$.

At $\mathrm{T}=4 \cdot 10^{8} \mathrm{~K},{ }^{15} \mathrm{O}(\alpha, \gamma){ }^{19} \mathrm{Ne}$ becomes the most important $\alpha$-capture reaction (besides $3 \alpha$ ), dominating ${ }^{14} \mathrm{O}(\alpha, \mathrm{p}){ }^{17} \mathrm{~F}$ up to $\mathrm{T} \sim 5 \cdot 10^{8} \mathrm{~K}$. The nuclear flow moves away from the valley of stability, towards the proton-drip line. This pattern is accompanied by an increase in the abundances of heavier species. Indeed, when $\mathrm{T}=1.5 \cdot 10^{9} \mathrm{~K}$, the most abundant species become ${ }^{18} \mathrm{Ne},{ }^{21,22} \mathrm{Mg}$ (from ${ }^{18} \mathrm{Ne}(\alpha, \mathrm{p}){ }^{21} \mathrm{Na}(\mathrm{p}, \gamma){ }^{22} \mathrm{Mg}$ ), ${ }^{25} \mathrm{Si},{ }^{28} \mathrm{~S}-{ }^{28} \mathrm{P},{ }^{33} \mathrm{Ar}-{ }^{33} \mathrm{Cl}$, and ${ }^{37} \mathrm{~K}$, the first isotope that achieves an abundance of $10 \%$ by mass. The ${ }^{4} \mathrm{He}$ abundance has already decreased below $1 \%$ by mass, hence reducing the extend of $\alpha$-capture reactions. At this stage, the flow has reached ${ }^{64} \mathrm{Ge}$. Shortly later, the envelope achieves peak temperature, $\mathrm{T}_{\text {peak }}=1.7 \cdot 10^{9} \mathrm{~K}$. The most abundant isotope (except for $\mathrm{H}$ ) becomes first ${ }^{54} \mathrm{Ni}$, and later, ${ }^{64} \mathrm{Ge}$ and ${ }^{68} \mathrm{Se}$. During the 
subsequent cooling phase, the nuclear flow is dominated by a cascade of $\beta$ decays (see Fig. 1, bottom panel).
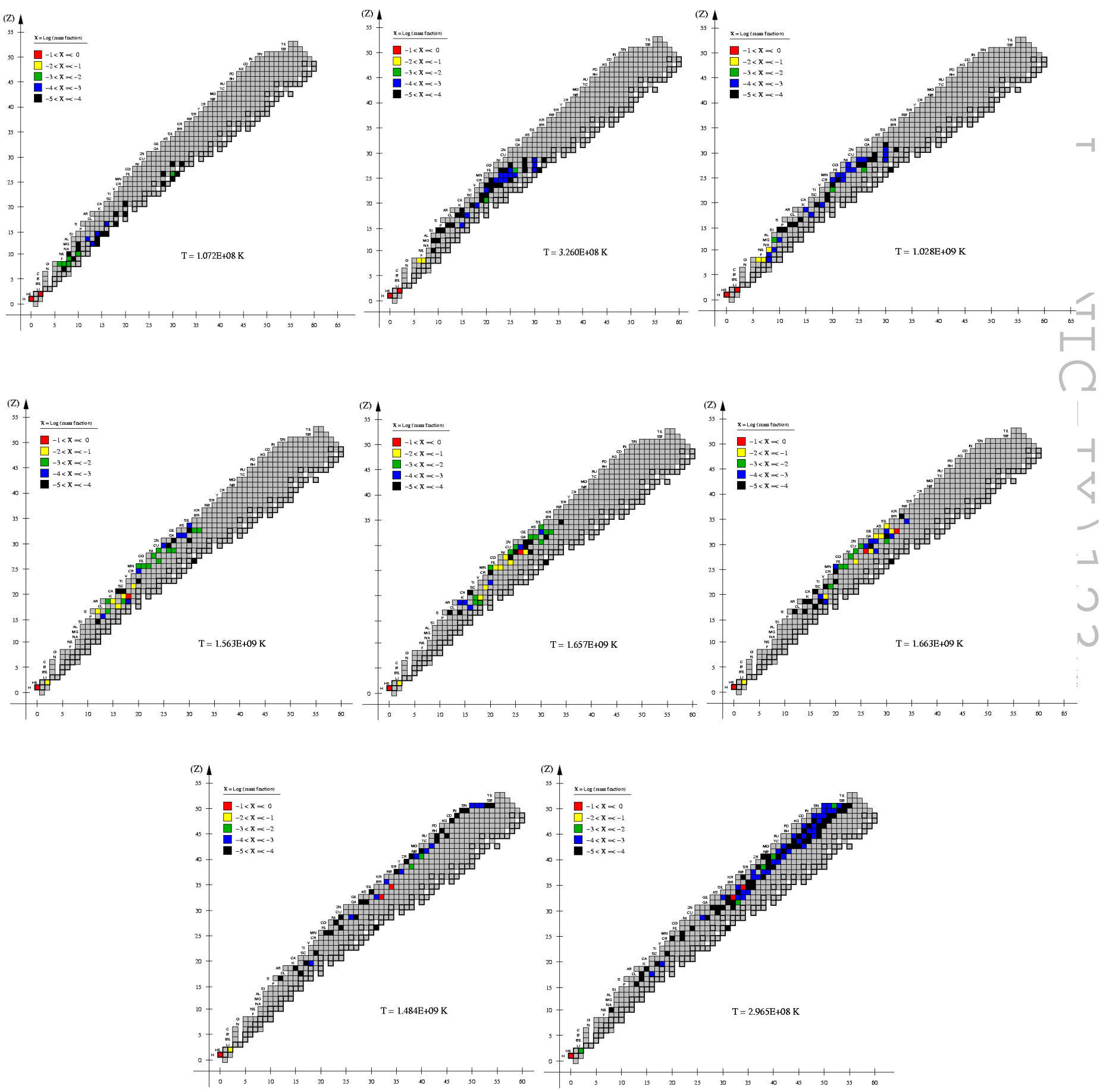

Fig. 2: Distribution of abundances by mass at the innermost shell of the accreted envelope for different stages of the TNR during a typical X-ray burst. 
The final composition of the envelope, which is not ejected by the TNR, is essentially made of elements of masses $\mathrm{A}=60-70$, mainly ${ }^{64} \mathrm{Zn}$ (originally as ${ }^{64} \mathrm{Ge}$, and ${ }^{64} \mathrm{Ga}$ ), and ${ }^{68} \mathrm{Zn}$ $\left({ }^{68} \mathrm{Se}\right)$, with traces of ${ }^{76} \mathrm{Se},{ }^{80} \mathrm{Kr}$, and ${ }^{102} \mathrm{Pd}$.

It is worth noting that details of the specific nucleosynthetic path usually suffer from nuclear uncertainties. In order to improve our knowledge on this matter, reaction rates and effective lifetimes of the 'waiting point' nuclei, as well as mass measurements at the main rpprocess path are required.

\section{References}

[1] J. L. Fisker, F.-K. Thielemann, \& M. Wiescher, The nuclear reaction waiting points: ${ }^{22} \mathrm{Mg},{ }^{26} \mathrm{Si},{ }^{30} \mathrm{~S}$, and ${ }^{34} A r$, and bolometrically double-peaked type I X-ray bursts, ApJ 608 (2004), L61.

[2] T. Hanawa, D. Sugimoto, \& M.-A. Hashimoto, Nucleosynthesis in explosive hydrogen burning and its implications in ten-minute interval of X-ray bursts, PASJ 35 (1983), 491.

[3] J. José, J., \& M. Hernanz, Nucleosynthesis in classical novae: CO vs. ONe white dwarfs, ApJ 494 (1998), 680.

[4] O. Koike, et al., Final products of the rp-process on accreting neutron stars, ApJ 603 (2004), 242.

[5] O. Koike, et al., Rapid proton capture on accreting neutron stars -effects of uncertainty in the nuclear process, A\&A 342 (1999), 464.

[6] H. Schatz, et al., The rapid proton process ashes from stable nuclear burning on an accreting neutron star, ApJ $\mathbf{5 2 4}$ (1999), 1014.

[7] H. Schatz, et al., The endpoint of the rp-process on accreting neutron stars, Nucl. Phys. A688 (2001), 150 .

[8] R. E. Taam, et al., Succesive X-ray bursts from accreting neutron stars, ApJ 413 (1993), 324.

[9] R. E. Taam, S. E. Woosley, \& D. Q. Lamb, The effect of deep hydrogen burning in the accreted envelope of a neutron star on the properties of X-ray bursts, ApJ 459 (1996), 271.

[10] R. K. Wallace, \& S. E. Woosley, S.E., Explosive hydrogen burning, ApJS 45 (1981), 389.

[11] R. K. Wallace, \& S. E. Woosley, Nuclear physics problems for accreting neutron stars, in High Energy Transients in Astrophysics, S. E. Woosley (ed.), New York: AIP, (1984), p. 319.

[12] S. E. Woosley, \& T. A. Weaver, Repeated thermonuclear flashes on an accreting neutron star, in High Energy Transients in Astrophysics, S. E. Woosley (ed.), New York: AIP, (1984), p. 273.

[13] S. E. Woosley, et al., Models for type I X-ray bursts with improved nuclear physics, ApJS 151 (2004), 75.

[14] M. Zingale, et al., Helium detonations on neutron stars, ApJS 133 (2001), 195. 